\title{
Biosimilars in dermatology
}

\author{
Małgorzata Mazur ${ }^{1}$, Karolina Olek-Hrab ${ }^{1}$, Jacek Karczewski², Ewa Teresiak-Mikołajczak ${ }^{1}$, Zygmunt Adamski ${ }^{1}$
}

${ }^{1}$ Department of Dermatology and Venereology, Poznan University of Medical Sciences, Poznan, Poland

Head of the Department: Prof. Zygmunt Adamski MD, PhD

2Department of Biology, Poznan University of Medical Sciences, Poznan, Poland

Head of the Department: Prof. Krzysztof Wiktorowicz MD, PhD

Postep Derm Alergol 2015; XXXII (5): 384-387

DOI: $10.5114 /$ pdia.2014.44026

\begin{abstract}
Over the last decade the availability of biological drugs for the treatment of psoriasis vulgaris, psoriatic arthritis and many other inflammatory diseases has revolutionized the treatment of these diseases around the world. Due to the high cost of therapy, the search has started for biosimilars. In dermatology the greatest interest in biosimilar medicines concerns inhibitors of tumor necrosis factor $\alpha$ (TNF- $\alpha$ ), for use in the treatment of psoriasis vulgaris and psoriatic arthritis (infliximab, etanercept, adalimumab). The most important element of the safety of biologicals is their immunogenicity. Therefore, when discussing biosimilars, attention needs to be paid to the dangers of their immune activity. In view of the fact that the drugs contain and aggregates, produced by living organisms or cultures of living cells, they cannot be compared in any way to low molecular weight synthetic generics (called generics). Biosimilars are authorized for use in patients and treated as equivalent to the reference medicine only after passing a number of studies and assessments. As it is well known, the development of medicine and pharmacology is extremely intense, and the market in biological medicine is developing much faster than that of all other drugs, which underlines their important role in modern medicine. Currently, the subject of biosimilars is one of the most important challenges and topics of discussion around the world, including pharmacovigilance and legal and economic regulatory standards. It seems inevitable that biosimilar products will be introduced for the treatment of diseases with indications corresponding to the original product on which they are based.
\end{abstract}

Key words: biosimilars, immunogenicity, psoriasis.

\section{Introduction}

Over the last decade the availability of biological medicines for the treatment of psoriasis, psoriatic arthritis and many other inflammatory diseases has revolutionized the treatment of these afflictions around the world. However, the high cost of treatment has significantly reduced the availability of treatment, restricting treatment to only a narrow group of the most seriously ill patients. That is why there is so much interest in biosimilars, the introduction of which into therapy would be a cheaper alternative to the treatment of a larger group of patients. In dermatology the greatest interest associated with biosimilars concerns tumor necrosis factor $\alpha$ (TNF- $\alpha$ ) inhibitors, used in the treatment of psoriasis and psoriatic arthritis (infliximab, etanercept, adalimumab). Biological medicines were registered for the treatment of psoriatic arthritis in 2002 (etanercept) and in 2005 (infliximab, adalimumab). In the case of psoriasis these were: 2004 - etanercept, 2006 - infliximab, 2008 - adal- imumab. The final biological preparation, blocking IL$12 / 23$, was ustekinumab, which was registered for the treatment of psoriasis in 2009. Many recent epidemiological studies have shown the influence of treatment with anti-TNF- $\alpha$ on body mass of patients with psoriasis. Ustekinumab will then have a significant influence on decreasing the production of cytokines, which are important for metabolism and body mass [1]. A biosimilar of etanercept is no longer sold in China or Colombia, and several biotech companies in Asia are in the process of obtaining biosimilars for anti-TNF inhibitors [2, 3].

\section{What is a biosimilar?}

The definition of a biosimilar medicine comes directly from the English translation of the term 'biosimilar drugs', which is used in the European Union, and explains that the product is biologically similar to a drug already registered and authorized for marketing in the European Union. The original product is referred to as the "refer-

Address for correspondence: Małgorzata Mazur MD, PhD, Department of Dermatology and Venereology, Poznan University of Medical Sciences, 49 Przybyszewskiego St, 60-355 Poznan, Poland, phone: +48 600250 215, e-mail: doktoressa@gmail.com Received: 12.03 .2014 , accepted: 5.05.2015. 
ence medicinal product" [4]. Biosimilars are produced by other manufacturers after the expiry of the patent right of the original medicines, which takes away the company's ownership right of the biological medicines [4]. The European Medicines Agency (EMA) emphasizes that both the biosimilar and the original product must have the same safety and efficacy profile, and present the same indications for use. In order to obtain the abovementioned information on a biosimilar medicine, it must undergo a thorough comparative study with the reference medicine, including the quality, safety and efficacy of the product. Finally, the product can be approved for use for the same indications as the reference drug. The first biosimilars accepted by the European Agency for the European Medicines Agency (EMA) in 2006 for use were preparations of recombinant human growth hormone - Omnitrope (original drug - Genotropin) and Valtropin (original drug - Humatrope) [5]. Professor Grieb points out that the term 'biosimilars' is not an accurate term to describe this kind of preparation, because in Poland this word is associated with something less valuable: a replacement compared to a chocolate-like product [4]. For comparison, in the U.S. these preparations are called 'follow-on biologics', which can be explained as 'bio-successive drug' [4, 6]. Biosimilars are produced using biotechnological technology or can come from natural sources, and at the same time must be sufficiently similar to the original product in order not to deviate from its safety or efficacy profiles [7]. However, it is often emphasized that biosimilars, as protein molecules, cannot be completely identical to the original product. This is because biological drugs, due to their high molecular complexity, are very sensitive to even small changes in production processes, which may be associated with the occurrence of unforeseeable side effects in biosimilar products. The differences in impurities and/or degradation products may have serious consequences for the health of patients. Therefore, the process of a development programme for biological medicines, according to the EMA and the World Health Organization (WHO), is very demanding and very specific. The registration of a biosimilar requires a lot of evidence for the efficacy and safety of these substances, namely detailed documentation of the production process of a biosimilar medicine, data on the stability of the preparation, pre-clinical studies comparing the preparation of the original drug, evaluating the physical, chemical and biological characteristics of the active ingredient, at least one preclinical toxicological study using multiple doses on animals, a study assessing the pharmacokinetics and pharmacodynamics of the product, a clinical trial of the 'head-to-head' type with the use of a single dose of the preparation compared to the original medicine, the phase III 'head-to-head' clinical trial, evaluating the efficacy and safety of the medicine studied compared to the original formulation, immunogenicity studies for people, as well as the provision of a long-term pharma- covigilance plan, which is subject to strict monitoring. Such strict criteria must be met in order for the drug to be approved for use as a biosimilar medicine [8]. Also, the pharmaceutical form, power of use and way of administration of the biosimilar must be identical to the reference medicine. Biosimilars which may be authorized for the treatment of patients with multiple indications must go through the strict regulatory requirements for registration. In Europe the EMA developed, on the basis of significantly legitimate concerns, strict guidelines for the authorization of biosimilars that have been adopted for use in Canada and the USA. However, in contrast, the regulatory requirements are significantly reduced in developing countries, such as India, which are becoming the leading precursors of biosimilars in the world [9].

\section{Immunogenicity}

The most important element of the safety of biological preparations is their immunogenicity. Therefore, when discussing biosimilars one needs to pay attention to the fear of their immune activity. This happens because any given protein can result in the formation of antibodies. Immune responses after administration of the drug can be very diverse, and include both their own reactions (e.g. anaphylaxis)and late responses (e.g. serum sickness) or the formation of neutralizing antibodies [10, 11]. Some immune reactions may occur even many years after the start of treatment, which is why today we are not able to predict late side effects of the biological treatment, including those of the original product. Biological medicines, including their biosimilar equivalents, are typically administered parenterally, and are particularly sensitive to storage conditions, which must also be highlighted during the assessment of the above-mentioned preparations [10]. Immunogenicity studies of biosimilars are required before launching them on the market, and must be equivalent to the safety of the original product.

\section{Biosimilars and generics}

In view of the fact that biological drugs contain active substances with the structure of biological polymers and aggregates, produced by living organisms or cultures of living cells, they cannot be compared to any of the low synthetic generics [4]. Generics are formed on the basis of the known chemical structure of the original preparation, which at the same time strictly regulates its properties. Therefore, prior to the registration of this type of product it is considered that one must carry out clinical tests [4]. As we can see, the main difference between the generic drug and a biosimilar explains a completely different approach to the possibility of biosimilar registration, subject to many constraints. For comparison, the biological medicine called 'interferon b' is characterized by a mass of particles of 19000 D, while the acetylsalicyl- 
ic acid molecule has a molecular mass of only $180 \mathrm{D}[10$, 12]. Opening up the world to biosimilars has stimulated considerable interest in this topic in the medical community. As we know, every doctor and patient is accustomed to the use of generic medicines, both as external preparations and general ones, which does not bear the concerns in the conduct of therapy, and is often dictated by economic considerations, at the same time concerning good therapeutic effects. However, when it comes to biosimilars, after passing many tests and evaluations, the medicine can be approved for use in the case of patients treated as an equivalent to the reference medicine. All restrictions on the planned registration of biosimilars are extremely important and have a great clinical relevance for the doctor who prescribes the drug, and above all, for the safety of the patient being treated. According to researchers from Connecticut, Strober and partners, one must be aware of the safety aspects of future treatments in order to give a chance to a larger group of patients with psoriasis all over the world who do not have access to biological therapies, while fostering the development of biosimilars which would be admitted for the treatment of patients after passing all levels of the registration ladder [13]. It seems that such preparations that pass the strict registration ladder would be safely used in the indications of the reference preparation. It should be noted that so far only a few biosimilars have been approved for the European market, but in the near future we can expect new preparations. The first candidate for monoclonal antibody similars, submitted for registration in the European Union to the EMA, is a preparation manufactured by Celltrion, which is a biosimilar of infliximab. It is highly likely that new biosimilars will be launched in the future, which is directly related to the end of the patent protection of a number of original medicines. Patent protection, as we all know, lasts for only 20 years, and therefore companies will seek to introduce drugs resembling biosimilars which will be based on their original predecessors [4]. This mainly concerns the widespread use of monoclonal antibodies. The biggest fear is associated with the immunogenicity of biosimilars since it is known that even the smallest detail in the production of different formulations can cause variations in many parameters. An example was the production of interferon $\beta 1 n$ by Biogen Idec (USA), which was originally created from the cell line of a Chinese hamster by Bioferon Biochemische Substanzen GmbH \& Co (Germany) [14].

\section{Biosimilars in dermatology}

Monoclonal biosimilar antibodies have been registered in India and South Korea [15]. The EMA approved the first applications for two monoclonal biosimilar antibodies which replace infliximab. The patent for infliximab is due to expire in Europe between 2013 and 2015. The first application was filed by Celltrion. This preparation,
Inflectra, is no longer sold in South Korea (CT - P13, Remsima) [16]. Before applying, the preparation underwent many tests required by the EMA and WHO for biosimilar agents, including the phase I clinical trial (PlanetAS) - a randomized, multicentre, parallel study with a double-blind trial, carried out in a group of patients with ankylosing spondylitis, which showed the equivalence of safety and efficacy in comparison with infliximab [17]. Another phase III clinical trial (PlanetRA) - a randomized, multicentre trial, parallel with the double-blind trial, was carried out in a group of patients with rheumatoid arthritis [18].

During the concomitant treatment with the tested drug and methotrexate, the safety and efficacy of the preparation was estimated in relation to the reference drug. To sum up, if all recommendations (prescreening, laboratory and pregnancy tests, adequate doses, optimal route of administration and folic acid supplementation) are fulfilled, MTX seems to be a safe, effective and relatively cheap treatment option in all forms of moderate-to-severe psoriasis, including psoriasis arthritis [19].

Inflectra is produced by Alvogen and Remsima by Celltrion concerns. Inflectra and Remsima are indicated in the treatment of rheumatoid arthritis, ankylosing spondylitis, psoriatic arthritis, colitis ulcerosa and Crohn disease in adults and children above 6 years of age. The clinical trials conducted in the group of patients suffering from rheumatoid arthritis and ankylosing spondylitis revealed comparable efficacy of tested medications to Infliximab. The aforementioned drugs can also be safely use in the treatment of psoriatic arthritis. The dosage of the biosimilars of infliximab is the same as in case of the original drug. The biosimilars of infliximab are also registred in the treatment of moderate and severe type of plaque psoriasis. Another biosimilar drug of infliximab remains in clinical research (Reliance Life Sciences, India).

\section{Conclusions}

The etiopathogenesis of psoriasis has not been fully elucidated. Nowadays, psoriasis is perceived as a multifactorial disease of genetic origin, in which complex immunological disorders concerning specific and non-specific defense mechanisms play a key role [20, 21]. New treatment possibilities for psoriasis and other inflammatory diseases using biological medicines has given patients the opportunity to achieve remission in severe forms of the disease. As new drugs, they initially met the concerns, including the safety and efficacy of therapy. Now, the situation is repeated in relation to the biosimilars. But is seems fully justified that in situations supported by the irrefutable results of clinical trials and clear evidence of efficacy and safety in the above-mentioned measures, a change in mentality may be brought about. It should be emphasized that only preparations that successfully pass the requirements of the EMA and 
WHO will be able to give more patients a chance to undergo biological treatment, at the same time ensuring their safety.

As we all know, the development of medicine and pharmacology is extremely intense, and the market in biological medicines is developing faster than for all other drugs, which highlights their important role in modern medicine. Currently, the matter of biosimilars is one of the most important challenges and topics of discussion all over the world, including pharmacovigilance, regulatory, legal and economic standards. It seems inevitable that biosimilars will be introduced for the treatment of diseases with indications corresponding to their original products. At the same time, drug registration departments will need to ensure standards of safety in order to allow treatment using only the preparations tested, in accordance with the guidelines of the EMA and WHO. The expected reduction in costs of biological treatment using biosimilars in Europe is estimated to be 15-30\% as compared to the original drug, which certainly allows savings in carrying out therapy. Currently, the part of biosimilar preparations remaining or planned is in the phase III of clinical trials in the 'head-to-head' studies in many centres around the world, including Poland. It seems that only time and experience will clarify concerns about biosimilars.

\section{Conflict of interest}

The authors declare no conflict of interest.

\section{References}

1. Owczarczyk-Saczonek A, Placek W, Rybak-d'Obyrn J, et al. Influence of ustekinumab on body weight of patients with psoriasis: an initial report. Postep Derm Alergol 2014; 31: 29-31.

2. Kay J. Biosimilars: a regulatory perspective from America. Arthritis Res Ther 2011; 13: 112.

3. Rondon F, Bautista A, Salazar JC, et al. Etanar therapy in real-life patients with rheumatoid arthritis. Arthritis Rheum 2010; 62: 1811.

4. Grieb P. Compendium on biosimilar medicines. Forum Nefrol 2001; 3: 193-7.

5. Nau JY. Omnitrope, first "biosimilar" drug of the European Union. Rev Med Suisse 2006; 2: 1206.

6. DiMasi JA, Faden LB. Competitiveness in follow-on drug R\&D: a race or imitation? Nature Rev Drug Disc 2011; 10: 23-7.

7. Woodcock J, Griffin J, Behrman R, et al. The FDA's assessment of follow-on protein products: a historical perspective. Nat Rev Drug Discov 2007; 6: 437-42.

8. WHO Expert Committee on Biological Standarization: guidelines on evaluation of similar biotherapeutic products (SBPs), Oct 2009 (WHO/BS/09.2110).

9. Mody R, Varshney B, Patankar D. Understanding variations in biosimilars: correlation with risk and regulatory implications. Int J Risk Saf Med 2010; 22: 27-40.

10. Nowicki M, Zimmer-Nowicka J. Biopharmaceuticals original and biosimilars. Onkol Prakt Klin 2007; 3: 120-7.
11. Thorpe R, Wadhwa M. Protein therapeutics and their immunity. EJHP Practice 2006; 12: 17-9.

12. Karpusas M, Whitty A, Runkel L, et al. The structure of human interferon-beta: implications for activity. Cell Mol Life Sci 1998; 54: 1203-16.

13. Strober BE, Armour K, Romiti R, et al. Biopharmaceuticals and biosimilars in psoriasis: what the dermatologist needs to know. Am Acad Dermatol 2012; 66: 317-22.

14. Rudick RA, Simonian NA, Alam JA, et al. Incidence and significance of neutralizing antibodies to interferon beta-1a in multiple sclerosis. Multiple Sclerosis Collaborative Research Group (MSCRG). Neurology 1998; 50: 1266-72.

15. Beck A. Biosimilar, biobetter and next generation therapeutic antibodies. MAbs 2011; 3: 107-10.

16. Mullard A. Can next-generation antibodies offset biosimilar competition? Nat Rev Drug Discov 2012; 11: 426-8.

17. Park W. A randomized, double-blind, phase I study demonstrates equivalence in pharmacokinetics, safety and efficacy of CT-P13 and infliximab in patients with ankylosing spondylitis. Orraly presented at EULAR Annular Conference 2012 Abstract Session: Spondyloarthritis - clinical aspects and treatment. Abstract OP0143.

18. Yoo D. A randomized, double-blind, phase 3 study demonstrates clinical equivalence of CT-P13 and infliximab when coadministered with methotrexate in patients with active rheumatoid arthritis. Presented at EULAR Annular Conference 2012, Poster Tour F02: RA anti-TNF. Poster FRI 0143.

19. Dańczak-Pazdrowska A. Place of methotrexate in the treatment of psoriasis in the era of biologic agents. Postep Derm Alergol 2012; 29: 182-8.

20. Chomiczewska-Skóra D, Trznadel-Grodzka E, Rotsztejn H. Psoriasis as a disease associated with the immune system disorders. Centr Eur J Immunol 2013; 38: 129-33.

21. Nedoszytko B, Sokołowska-Wojdyto M, RuckemannDziurdzińska K, et al. Chemokines and cytokines network in the pathogenesis of the inflammatory skin diseases: atopic dermatitis, psoriasis and skin mastocytosis. Postep Derm Alergol 2014; 31: 84-91. 